\title{
Prediction of Mobility Entropy in an Ambient Intelligent Environment
}

\author{
Saisakul Chernbumroong, Ahmad Lotfi and Caroline Langensiepen \\ School of Science and Technology, Nottingham Trent University \\ Nottingham, NG11 8NS, United Kingdom \\ Email: \{saisakul.chernbumroong, ahmad.lotfi, caroline.langensiepen\}@ntu.ac.uk
}

\begin{abstract}
Ambient Intelligent (AmI) technology can be used to help older adults to live longer and independent lives in their own homes. Information collected from AmI environment can be used to detect and understanding human behaviour, allowing personalized care. The behaviour pattern can also be used to detect changes in behaviour and predict future trends, so that preventive action can be taken. However, due to the large number of sensors in the environment, sensor data are often complex and difficult to interpret, especially to capture behaviour trends and to detect changes over the long-term.
\end{abstract}

In this paper, a model to predict the indoor mobility using binary sensors is proposed. The model utilizes weekly routine to predict the future trend. The proposed method is validated using data collected from a real home environment, and the results show that using weekly pattern helps improve indoor mobility prediction. Also, a new measurement, Mobility Entropy (ME), to measure indoor mobility based on entropy concept is proposed. The results indicate ME can be used to distinguish elders with different mobility and to see decline in mobility. The proposed work would allow detection of changes in mobility, and to foresee the future mobility trend if the current behaviour continues.

\section{INTRODUCTION}

Global human population has a rapid growth and the rate of the elderly people is increasing in the population. The results published by United Nation is predicting that the number of the elderly people will be approximately 1.2 billion in 2025 [1]. The rate of the elderly in developed countries is about $28 \%$. It is projected that by 2050 , people aged 65-79 will constitute almost a third of the population of Europe, whilst the number of the oldest old (80+) will rise by $180 \%$ [2]. The majority of older adults prefer to remain in their homes for as long as possible, and nowadays technologies are available to help them live longer and independent lives in their own homes. One of these technologies is referred to as an Ambient Intelligent (AmI) environment, where multiple sensors are deployed in the home. These sensors include Passive Infrared (PIR), door switch, pressure mat, temperature sensor, flood sensor, smoke detector, etc. The data collected from the sensors can be used to monitor and detect the older adult's activities to provide security, support, and enhance independence living. For example, a kettle can be automatically switched on when a person gets up.

However, sensor data are often complex and difficult to interpret, especially to capture behavioural trends and to detect changes over a long-term period. The ability to detect human behaviour will allow personalized care, and improve the understanding of their behaviour. The behaviour pattern can also be used to detect changes in behaviour and predict future trends, so that preventive action can be taken. The understanding of human behaviour has gained significant attention from researchers. A number of studies have been conducted to capture and analyse human behaviour, ranging from short temporal resolution e.g. posture and movement transition [3], and activity recognition [4] to longer resolution such as longterm behaviour patterns [5]. Computational intelligence techniques such as neural networks [6], Hidden Markov Model [7], fuzzy logic [8], mixture model [5], etc. as well as semantic approaches [9], [10] have been used to model, learn, and discover behavioural patterns.

In this study, a model to predict the indoor mobility using binary sensors is developed. Unlike other works, the sensor data are converted into room transitions which are then used to capture the indoor mobility. We propose a prediction model by utilizing day of the week patterns in a neural network. The hypothesis is that humans generally have weekly routines, and that their behaviour pattern can be explained by the weekly pattern. The aim is to evaluate and compare the performance of the model using day of the week patterns and the models using previous indoor mobility values. We also propose a new measurement to measure the indoor mobility based on entropy.

This paper is organized in the following order; previous works in human behaviour prediction is reviewed in the next section followed by the explanation about the indoor mobility in Section III. Indoor mobility prediction is explained in Section IV followed by detailed explanation of indoor mobility prediction based on weekly pattern in Section $\mathrm{V}$ then the proposed mobility entropy is presented in Section VI. Results are presented in Section VII followed by discussion in Section VIII. Pertinent conclusions are drawn in Section IX.

\section{RELATED WORKS}

Various attempts have been made to understand human behaviour and to predict future events. Work by [6] studied two types of recurrent neural network models i.e. NARX, and Elman network, to predict the behaviour using occupancy sensors including PIR and door entry point sensors. In their method, the raw binary sensor data are converted into sensor start time and duration. The results of the study indicated that the NARX network's performance is better than the Elman network. They also compared the results with a moving average technique and found that NARX produced much better results. The results of this study indicated the powerful performance of NARX in time-series prediction.

Another common technique for time series detection and prediction is the Hidden Markov Model (HMM). It can be used 
to learn previous input sequences and estimate future state. An algorithm to detect behaviour patterns based on HMM is proposed in [7]. They used binary inputs to learn the behaviour of conference events. However, HMM suffers in performance terms when a large amount of data is used [6].

Clustering techniques have also been used to discover behaviour patterns. A study by [5] examined a motion sensor system to detect behavioural patterns. They applied mixture model methods on the time spent in one location to divide the data into clusters. The cluster are later compared to the person's activity log to determine the activities that matched the clusters. They found that the discovered clusters corresponded to sleeping, changing clothes, bathroom/toilet use, leaving/returning home, and meal preparation.

A method to recognize complex activity patterns using a probabilistic grammar approach is proposed in [9]. Images from a camera are used to determine the location of a person within an environment which had been divided into different regions such as dining, sink, stove, etc. The grammar is used to define activities e.g. cooking and the probability of action given the area sequence. The grammar defined must be general enough to allow multiple instances of an activity, but still be able to detect different actions. The grammar definition imposes restriction of specific locations over time. Their experimental results indicated that a good grammar and precise locations are essential in this technique. Visual sensors are also used in [11]. They proposed a method to learn behaviour patterns where information of the person's location is extracted and action is identified using k-Nearest Neighbour, then data mining techniques are used to discover the frequency behaviour pattern. The experiments demonstrated the use of their proposed method, although the studied scenarios are limited i.e. work scenario. However, the use of such visual sensors may not be acceptable in home monitoring application due to privacy issues. The alternative is to utilize non-visual sensors e.g. PIR to obtain location or events instead.

A framework is proposed in [10] to detect behavioural patterns to help dementia patients using Workflow Mining technology. The flow of activities are captured via an algorithm called the Workflow Instance Acceptor Algorithm. The algorithm shows how the flow of actions fits with the expected flow and if there is any difference, it is highlighted. Based on this information, the health professional can decide what motivation plan to apply to the patient. The advantage of this method is that it allows behaviour to be represented in a formal semantic way which helps in understanding the behaviour, especially for non-technical users. However, further analysis on the received information, experience, and expertise would be required to make informed decisions.

Studies shown that the older adults spend most of the day inactive [12]. It is encouraged that the older adults are engaged in physical activity to optimize their health outcomes as activity level has been shown to be associated with survival rate, independence, and quality of life [13]. Physical activity often diminishes as age increases, especially in older women [14]. Majority of the assessments are developed to measure the physical activity level. However, these measurements are mostly focused on the outdoor activities, only a few assessment are developed to measure indoor mobility at homes. For example, the nursing home life-space diameter (NHLSD) [15] is developed to measure indoor mobility in nursing home environment. The nursing home spaces are divided into different zones i.e. resident's room, in unit, out of unit, and out of facility. The measurement is based on how often between zones within 2-week period. Their results shows that NHLSD is associated with ADL. However, since NHLSD is developed on nursing home setting, it may not effectively assess the indoor mobility for home setting.

Another study [12] proposed a measurement to describe indoor life-space mobility at home (LSH). The LSH is calculated based on how far and how often a participant moved from a bedroom to four locations i.e. entrance, dining room, bathroom, and toilet, and whether a participant requires any assistance. The distances between each room are measured and each week the participants are asked the number of times they moved to each location, and if they needed any assistance. Higher LSH indicates greater indoor life-space mobility. The results of the study indicated that LSH reflects indoor life-space mobility and showed high correlation with basic activities of daily living e.g. feeding, sleeping, etc. which can be used to indicate a major decline in indoor activities and physical abilities. It will provide a useful assessment for older adults with difficulty in performing outdoor activities. The limitation of these approaches is that LSH and NHLSD are based on questionnaires as older persons are likely to forget what activities they have done over a long period.

In this study, the same concept as NHLSD and LSH are used such that the Indoor Mobility (IM) is calculated based on movement within homes. However, instead of using questionnaire, the AmI sensors are used to collect the movement information of an older adult. This allows movement data to be collected automatically to reduce human error. The aim is to capture the IM pattern and develop a model to predict the future IM. We also propose a new measurement which is used to measure the level of indoor mobility based on entropy. The result of the study would allow detection of changes in the person's indoor mobility, and to foresee the future trend if the current behaviour continues.

\section{INDOOR MOBILITY}

The indoor mobility is represented by the frequency of the movement from room to room. Given a home environment equipped with AmI sensors such as PIR, a person's transitions in home can be detected. For simplicity, we will consider only binary sensors. Given any binary sensor $S$ at location $l$, its value at time $t$ can be represented as:

$$
S_{t}^{l}= \begin{cases}0 & \text { OFF } \\ 1 & \text { ON }\end{cases}
$$

For any given time, a sequence of any sensor data can be written as $S^{l}=\left\{S_{0}^{l}, S_{1}^{l}, \ldots, S_{t}^{l}\right\}$ and the transition between rooms can be written as $T r=$ $\left\{\left(S^{l_{1}}, S^{l_{2}}\right)_{s t_{1}},\left(S^{l_{2}}, S^{l_{3}}\right)_{s t_{2}}, \ldots,\left(S^{l_{i}}, S^{l_{j}}\right)_{s t}\right\}$ where $i \neq j$ and $s t$ is the time the person enters the location $i$. Then, the IM is the total number of tuple in $T r$ between time $T 1$ and $T 2$.

$$
I M=\left|\left(S^{l_{1}}, S^{l_{2}}\right)_{s t_{1}}, \ldots,\left(S^{l_{i}}, S^{l_{j}}\right)_{s t}\right|_{T 1}^{T 2}
$$



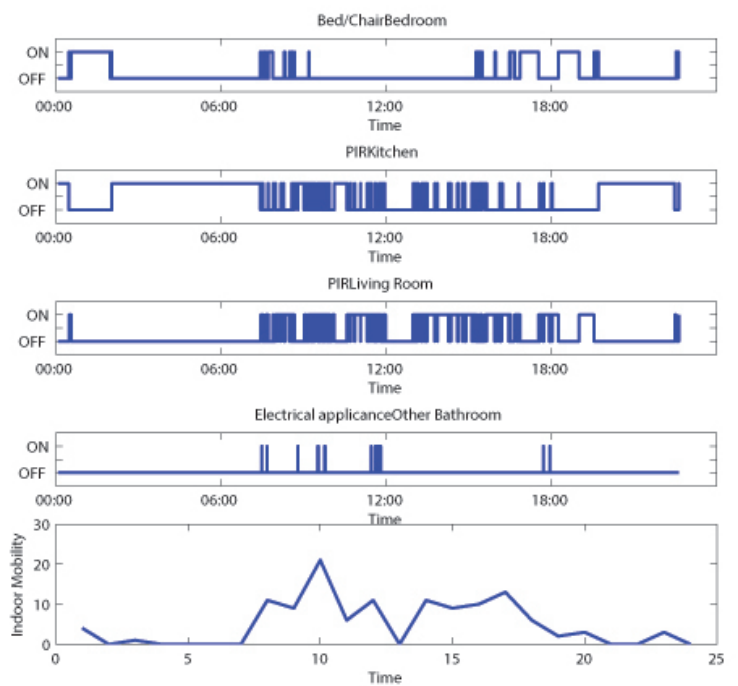

Fig. 1. An example of raw sensor data in various locations and the calculated indoor mobility over 24-hour period.

The data sets are collected from binary sensors installed in two single-occupancy homes. The data sets contain binary data from sensors including PIR, door contact switch, pressure sensor, and electrical appliance usage sensor. The information in the data sets include the date and time when the sensor is activated, sensor type, sensor value, and the location of the sensor. An example of a data is shown in Table I. The data sets are collected over a period of two months for each data set. The data is segmented using a 1-second interval. The sensor data are combined if they occur within the same second. For any sensor that is fired more than once within the second, the latest sensor value is used. Sometimes the time spend in the room detected by the sensor is very low, this is because a person is only passing through the room in order to go to another location. To handle this problem, the transition that occurs when the person spends at least 1 minute in the room is considered. In this study, the IM is calculated using 1-hour interval i.e. indoor mobility per hour. Fig. 1 shows the raw input and the IM calculated on a particular day.

\section{INDOOR MOBILITY PREDICTION}

In this study, neural network technique i.e. multi-layer perceptron (MLP) and recurrent network for IM prediction are investigated. A typical MLP is consisted of three layers including input, hidden, and output layers. The output is summation of the inputs and associated weights which can be written as:

$$
I M=f\left(\sum_{i}\left(w_{i} x_{i}\right)\right)
$$

TABLE I. AN EXAMPLE OF BINARY SENSOR DATA IN A DATA SET

\begin{tabular}{|l|c|c|c|}
\hline Date and time & Sensor type & Sensor value & Location \\
\hline 2004-10-01 00:22:15.093 & PIR & 1 & Master bedroom \\
2004-10-01 00:24:46.203 & PIR & 1 & Main bathroom \\
\hline
\end{tabular}

where $f$ is the activation function and is usually a sigmoid function.

A recurrent network uses the current input and the previous input sequences to predict the outputs. In particular, the model is developed using a recurrent dynamic network where the output is fed back to the input layer. The results of previous study [6] indicated that the recurrent network provide a good performance in predicting time-series data. To predict the future value of IM at time $t$, the following function is used:

$$
I M_{t}=f\left(I M_{t-1}, I M_{t-2}, \ldots, I M_{t-d}\right)
$$

where $d$ is the number of tapped delays. This network is referred to as a Nonlinear Autoregressive (NAR) network. If other time-series inputs, $x$, are used in the network, the future value of $I M_{t}$ can be rewritten as:

$$
\begin{gathered}
I M_{t}=f\left(I M_{t-1}, I M_{t-2}, \ldots, I M_{t-d},\right. \\
\left.x_{t-1}, x_{t-2}, \ldots, x_{t-d}\right)
\end{gathered}
$$

This network is referred as a Nonlinear Autoregressive with Exogenous Input (NARX).

\section{INDOOR MOBILITY PREDICTION BASED ON WEEKLY PATTERN}

A model to predict IM based on the day of the week pattern is proposed. The day of the week pattern is constructed using data collected over a period of $l$ days. For example, to predict IM at time $t$, the previous data of $l$ days are used to build day of the week patterns, $\phi_{t}^{D}$. The day of the week pattern is calculated from the statistical features including minimum, maximum, quartile at $25 \%, 50 \%$, and $75 \%$, upper and lower whisker, and upper and lower notch of a particular day of the week and hour e.g. Monday $8 \mathrm{am}$. The pairwise correlation shows that quartile at $75 \%$, upper whisker, and maximum are highly correlated which is not surprised as upper whisker is calculated from $w h i=$ minq $3+1.5 I Q R$, maximum. Similarly, quartile at $25 \%$, lower whisker, and minimum are highly correlated as lower whisker can be calculated from $w l o=\max q 1-1.5 I Q R$, minimum. Also there is a strong correlation between lower notch and quartile at $50 \%$ and upper notch and quartile at $50 \%$ as lower and upper notch can be calculated from $n l o=q 2+\frac{1.57 I Q R}{\sqrt{n}}$ and $n h i=q 2-\frac{1.57 I Q R}{\sqrt{n}}$, respectively. Therefore, it is possible to reduce some variables for prediction. Consequently, the weekly pattern is represented using quartile at 25\%, 50\%, and 75\%, and Median Absolute Deviation (MAD):

$$
\phi_{t}^{D}=\{Q 25, Q 50, Q 75, M A D\}
$$

In our model, the input is the pattern of the relevant day of the week. For example, to predict IM on Monday, the Monday pattern will be used. All neural network models contain three layers i.e. input, hidden, and output layers.

For MLP model, the predicted IM is based on weekly patterns only: 


$$
I \hat{M}_{t}^{D}=f\left(\phi_{t}^{D}\right)
$$

For NAR model, the predicted IM is based on previous IM only;

$$
I M_{t}^{D}=f\left(I M_{t-1}^{D}, I M_{t-2}^{D}, \ldots, I M_{t-\alpha}^{D}\right)
$$

Finally, in NARX model, the predicted IM at time $t$ can be represented as a function of a previous pattern of the day of the week and previous IM values:

$$
\begin{aligned}
I \hat{M}_{t}^{D}= & f\left(\phi_{t-1}^{D}, \phi_{t-2}^{D}, \ldots, \phi_{t-\alpha}^{D}\right. \\
& \left.I M_{t-1}^{D}, I M_{t-2}^{D}, \ldots, I M_{t-\alpha}^{D}\right)
\end{aligned}
$$

where $D$ is day of the week, e.g. Monday, Tuesday, etc., $\phi_{t}^{D}$ represents the pattern of the day $D$ at time $t, I M_{t}^{D}$ represents the indoor mobility at time $t$, and $\alpha$ is the number of delays in hours. For example, $\phi_{t}^{M o n}$ represents the Monday pattern at time $t$.

\section{INDOOR MOBILITY MEASUREMENT BASED ON ENTROPY}

A new measurement called Mobility Entropy (ME) is also proposed to measure the human's indoor mobility level. ME can be used to compare mobility level between persons and to use it as an indicator to detect changes in IM. The entropy is a concept introduced in thermodynamic to capture the amount of order, disorder, chaos in thermodynamic system [16]. It has been applied in other domains, for example, the concept of entropy is used to model spatial learning [17]. In this work, a person who is highly active will have high indoor mobility frequency and high dispersal throughout the day (high entropy) comparing to a person who is less active (low entropy). Utilizing this concept, the mobility entropy can be calculated from the entropy of frequency $\left(H_{\text {frequency }}\right)$ and entropy of dispersal $\left(H_{\text {dispersal }}\right)$ of the mobility over a week period. The entropy of frequency explains the frequency a person moves in an hour, and the entropy of dispersal explains the dispersal of movement over the day. Given IM is the frequency of indoor mobility per hour, the mobility of 1 week period can be written as $I M W=\left\{I M_{1}, I M_{2}, \ldots, I M_{7 \times 24}\right\}$. The mobility entropy can be calculated as:

$$
H_{M E}=H_{\text {Frequency }}+H_{\text {Dispersal }}
$$

where $H_{\text {Frequency }}=\ln \left(\sum I M W\right)$ and $H_{\text {Dispersal }}=$ $\ln (|I M W>0|)$.

\section{A. Validation procedure}

The performance is calculated using Mean Squared Error (MSE). Given $m$ time series, the error can be calculated as:

$$
M S E=\frac{1}{m} \sum_{i=1}^{m}\left(I M_{i}-I \hat{M}_{(i)}\right)^{2}
$$

where $I M_{(i)}$ is the indoor mobility at time $i$ and $I \hat{M}_{(i)}$ is the predicted mobility at time $i$.

The experiments are carried out using 7 days for training, 7 days for validation, 7 days for testing. Another 7 days of data is used as additional testing data for long-term prediction. More experiments are conducted with four different lead times to build day of the week patterns i.e. 14, 21, 28 and 35 days. The experiments were repeated for 5 times, hereafter this will be referred to as 5 folds. For each model setting i.e. number of hidden nodes, number of delay, number of lead times, 10 networks were built and the results reported are the average performances. Table II gives an example of how the data is separated for training and testing for a lead time of 14 days. The number in the table represents the day e.g. day 01/09$04 / 10$ are used for training.

The results are compared using statistical tests at $95 \%$ confident interval. The data is first checked for its normality using Shapiro-Wilk. If the data had a normal distribution, then a paired T-test is applied, otherwise Wilcoxon Signed Ranks Test is used.

\section{RESULTS}

\section{A. Indoor mobility behaviour based on day of the week pattern}

Fig. 2 shows the indoor mobility (IM) of a subject based on a month's data. From the graph, it can be seen that the person normally wakes up at 8 am and goes to bed at $11 \mathrm{pm}$. The graph also shows that the IM during day time $(12-3 \mathrm{pm})$ has a high variation. The behaviour pattern also shows that the subject is normally active during $6-9 \mathrm{pm}$.

Next, results of the day of the week pattern e.g. Monday, Tuesday, etc. based over 1 month (e.g. 4 Mondays) are investigated. According to the graph, there is no IM during $11 \mathrm{am}$ to $3 \mathrm{pm}$ on Monday. When inspect the data, it is found that the subject is resting in the living room during that period of time. Based on the behaviour patterns, it can be seen that the subject rests between $12-2 \mathrm{pm}$ on Wednesday and during $1-2$ $\mathrm{pm}$ on Thursday. The patterns show that the subject generally becomes active in the evening (from $4 \mathrm{pm}$ ) and is more active on Friday, Saturday, and Sunday. The patterns also show that the subject has a good sleep pattern in general. However, the pattern shows that the subject had disturbed nights mostly on Thursday, and Friday.

\section{B. Prediction models}

The aim of the experiment is to compare the performance of the model using only previous indoor mobility values (NARIM), the model using previous IM values and day of the week patterns (NARX-IM), and the model using only day of the week patterns (MLP-IM). First, the experiments are carried out to determine the appropriate number of hidden nodes and

TABLE II. AN EXAMPLE OF HOW THE DATA ARE SEPARATED FOR BUILDING DAY OF THE WEEK PATTERN $\left(\phi^{D}\right)$, TRAINING (TR), VALIDATION (VAL), TESTING (TE), AND ADDITIONAL TESTING (TE1).

\begin{tabular}{|c|c|c|c|c|c|}
\hline Fold & $\phi^{D}$ & TR & VAL & TE & TE1 \\
\hline 1 & $01 / 09-04 / 10$ & $5 / 10-11 / 10$ & $12 / 10$ & $13 / 10-19 / 10$ & $20 / 10-26 / 10$ \\
1 & $15 / 09-04 / 10$ & $5 / 10-11 / 10$ & $12 / 10$ & $13 / 10-19 / 10$ & $20 / 10-26 / 10$ \\
2 & $02 / 09-05 / 10$ & $6 / 10-12 / 10$ & $13 / 10$ & $14 / 10-20 / 10$ & $21 / 10-27 / 10$ \\
2 & $16 / 09-05 / 10$ & $6 / 10-12 / 10$ & $13 / 10$ & $14 / 10-20 / 10$ & $21 / 10-27 / 10$ \\
\hline
\end{tabular}



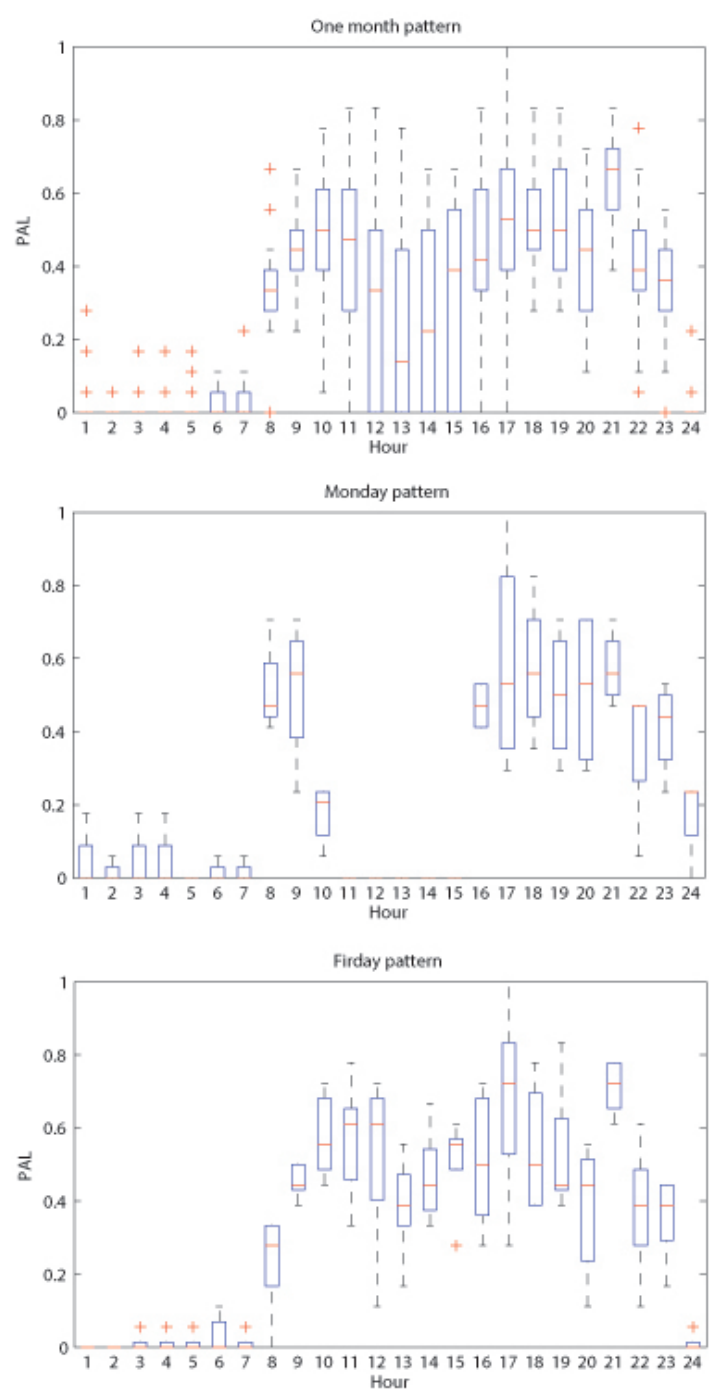

Fig. 2. Box-plot representing behavioural pattern based on IM for a duration of one month.

delays. The networks are constructed using 5 to 50 hidden nodes and 1 to 10 hours delay. For each setting i.e. $H$ hidden nodes, $\alpha$ delays, 10 models are built. The networks are trained using the Levenberg-Marquardt algorithm and tangent sigmoid is used as an activation function. The results are based on the average performance on the test set and additional test set. Experiments are also carried out to evaluate the effect of using different periods of lead time for the day of week patterns i.e. $l=14,21,28$ and 35 days. The performances of the models based on the their most optimum setting on different lead times are shown in Table III and Table V. Statistical tests are applied to see if the differences in performances are significant. The statistical results when comparing different models are shown in Table IV and Table VI, for data set 1 and 2, respectively.

Using the most optimum setting obtained from earlier experimental results, the additional test data are applied to evaluate the models' performances in multi-step prediction. The performance of the models in predicting indoor mobility in 1 day and 7 days advance are shown in Table VII and Table IX. The results are compared using statistical tests and the results are shown in Table VIII and Table X.

In terms of training time, in general the training time increases when the number of hidden nodes and delays increases. The training time of MLP models are less than NARX since fewer number of inputs are used, however this is not significant.

\section{Mobility entropy validation}

To validate the proposed indoor mobility, the simulated data which represent different mobility characteristic are used. The data for two case studies are simulated. The first case study is used to demonstrate the mobility entropy of four types of elders - a person with high frequency of mobility per hour and high dispersal of mobility throughout the day (profile 1), a person with low frequency and low dispersal of mobility (profile 2), a person with high frequency and low dispersal of mobility (profile 3 ), and a person with low frequency and high dispersal of mobility (profile 4). The data is generated randomly from a normal distribution where a mean of 0.8 mobility level is used for high frequency and 0.2 is used for low frequency. The probability of a person performing movement between rooms are generated from a normal distribution where $70 \%$ chance of movement occurring is used for high dispersal and $30 \%$ for low dispersal. Fig. 3 shows the mobility entropy of elders with different profiles in 1-week period. Fig. 4 shows the graph of mobility entropy over a 7-week period.

Another case study is used to evaluate the mobility entropy to detect the decline in mobility. The data is generated randomly to represents two elders' profiles i.e. a person who has high frequency then decline to low frequency of mobility (change from 0.8 mobility level to 0.2 ), and the same person who decline to low dispersal of mobility throughout the day (change from $70 \%$ chance of performing mobility to $30 \%$ ). Fig. 5 shows the mobility entropy when elders have decline in their indoor mobility. The data is generated randomly from a normal distribution for a 14-week period where the changes occur from week $8^{\text {th }}$ onwards.

TABLE VII. PERFORMANCES' OF THE MODELS IN 1-DAY AND 7-DAY AHEAD PREDICTION USING ADDITIONAL TEST DATA FOR DATA SET 1.

\begin{tabular}{|l|c|c|}
\hline Model & 1-day ahead prediction & 7-day ahead prediction \\
\hline NAR-IM & $0.0291 \pm 0.0102$ & $0.0356 \pm 0.0078$ \\
NARX-IM-14 & $0.0270 \pm 0.0089$ & $0.0258 \pm 0.0017$ \\
NARX-IM-21 & $0.0269 \pm 0.0090$ & $0.0252 \pm 0.0017$ \\
NARX-IM-28 & $0.0239 \pm 0.0084$ & $0.0231 \pm 0.0028$ \\
NARX-IM-35 & $0.0229 \pm 0.0073$ & $0.0219 \pm 0.0033$ \\
MLP-IM-14 & $0.0197 \pm 0.0095$ & $0.0193 \pm 0.0013$ \\
MLP-IM-21 & $0.0151 \pm 0.0076$ & $0.0166 \pm 0.0009$ \\
MLP-IM-28 & $0.0144 \pm 0.0074$ & $0.0168 \pm 0.0013$ \\
MLP-IM-35 & $0.0229 \pm 0.0073$ & $0.0219 \pm 0.0033$ \\
\hline
\end{tabular}

TABLE IX. PERFORMANCES' OF THE MODELS IN 1-DAY AND 7-DAY AHEAD PREDICTION USING ADDITIONAL TEST DATA FOR DATA SET 2.

\begin{tabular}{|l|c|c|}
\hline Model & 1-day ahead prediction & 7-day ahead prediction \\
\hline NAR-IM & $0.0494 \pm 0.0145$ & $0.0640 \pm 0.0173$ \\
NARX-IM-14 & $0.0336 \pm 0.0119$ & $0.0314 \pm 0.0053$ \\
NARX-IM-21 & $0.0360 \pm 0.0129$ & $0.0323 \pm 0.0026$ \\
NARX-IM-28 & $0.0338 \pm 0.0174$ & $0.0308 \pm 0.0037$ \\
NARX-IM-35 & $0.0273 \pm 0.0104$ & $0.0292 \pm 0.0034$ \\
MLP-IM-14 & $0.0268 \pm 0.0100$ & $0.0277 \pm 0.0019$ \\
MLP-IM-21 & $0.0218 \pm 0.0103$ & $0.0259 \pm 0.0034$ \\
MLP-IM-28 & $0.0197 \pm 0.0063$ & $0.0237 \pm 0.0014$ \\
MLP-IM-35 & $0.0199 \pm 0.0061$ & $0.0229 \pm 0.0017$ \\
\hline
\end{tabular}



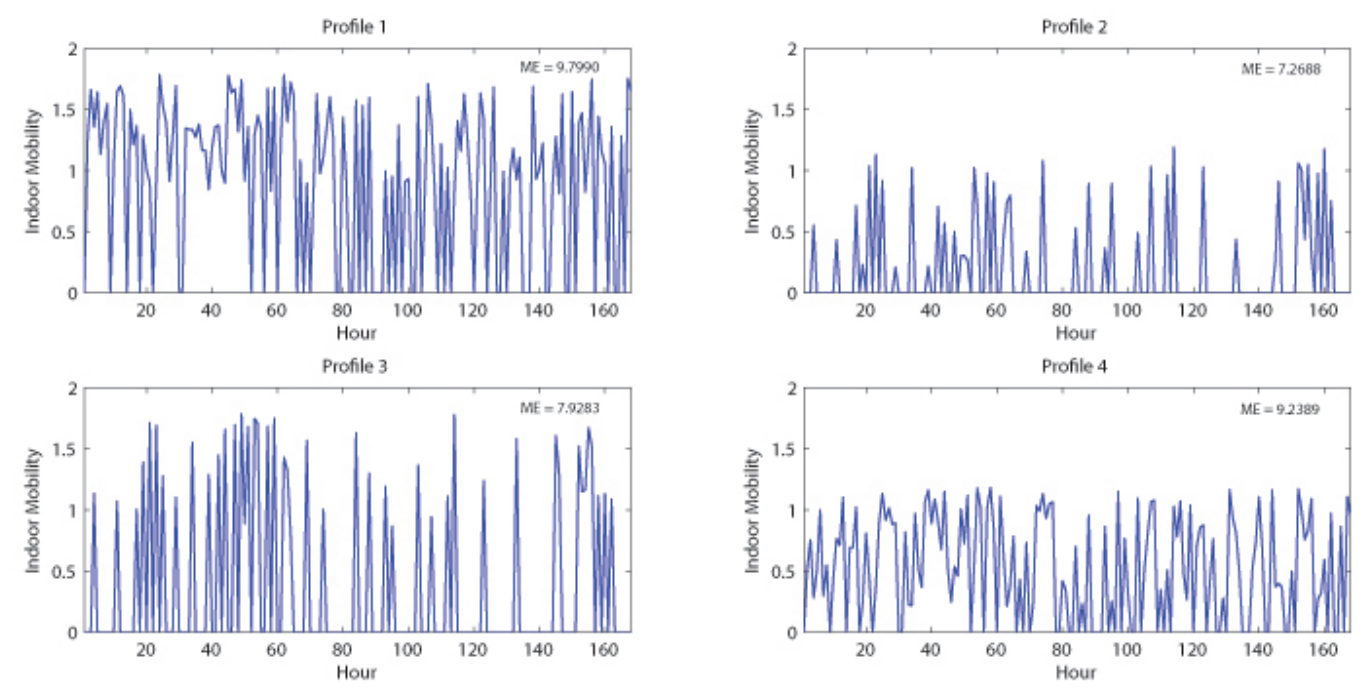

Fig. 3. The indoor mobility of elders with different profiles over a 7-week period and their mobility entropy.

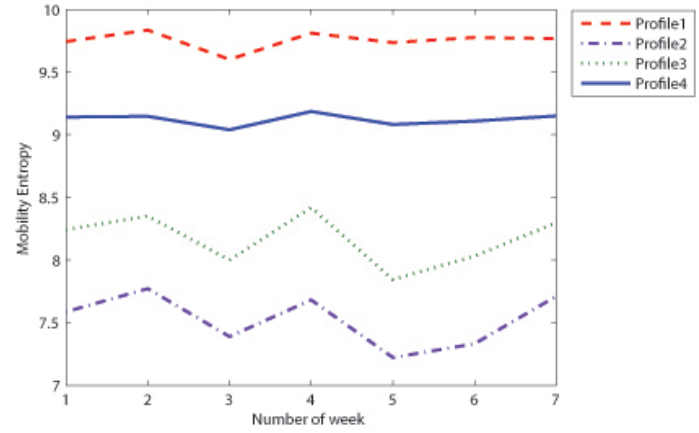

Fig. 4. The Mobility Entropy of elders with different indoor mobility profiles over a 7-week period. Profile 1 is a person with high frequency of mobility per hour and high dispersal throughout the day, Profile 2 is a person with low frequency and low dispersal, Profile 3 is a person with high frequency and low dispersal, and Profile 4 is a person with low frequency and high dispersal.

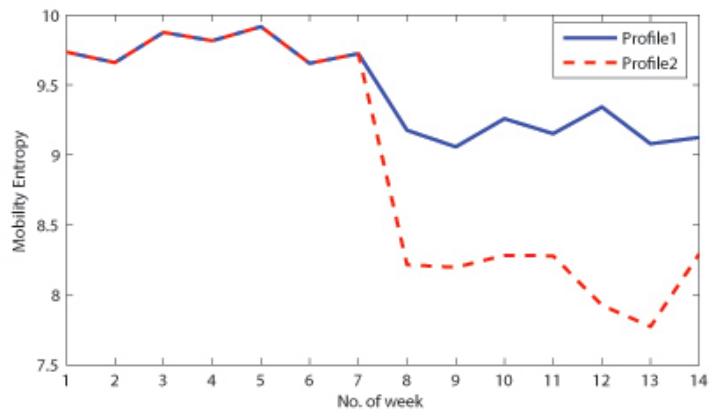

Fig. 5. The Mobility Entropy of elders who have decline in their indoor mobility over a 14-week period. Profile 1 is a person with high frequency of mobility per hour then decline to low frequency, Profile 2 is a person with high dispersal of mobility throughout the day then decline to low dispersal.

\section{DISCUSSION}

The results of the study show that although humans behave differently each day, it is possible to capture the behaviour using weekly patterns. By utilizing the indoor movement, we can see the behaviour trend of a person, and detect abnormal activity via behaviour pattern statistics and visual inspection. In this study, we captured the indoor mobility (IM) using AmI sensors and proposed to use the weekly pattern to predict future indoor mobility. The results show that MLP models are better than NAR and NARX models with statistical significance for both data sets. In data set 2, NARX models are better than NAR model. This results imply that using weekly patterns help in indoor mobility prediction. This is because weekly pattern captures information of $l$ days indoor mobility. However, using weekly patterns with previous weekly patterns (NARX models) does not guarantee to improve the prediction performances. From the correlation analysis, we have found that there is no significant relationship between previous IM values and current IM values, and previous weekly pattern and current weekly pattern. Therefore, by using the previous IM or weekly pattern values might not improve the prediction performance.

Regarding the effect of using different lead times, we have found that in general there is no statistical significant difference in NARX models, although the results show that using NARX with 35-day lead time is the most optimum for data set 1 and NARX with 28-day for data set 2. In MLP models, using different lead times has significant effect on the prediction performances and the results showed that using MLP with 28day lead time is the most optimum for data set 1 and 35-day for data set 2. Based on these results, it is suggested that using the lead time of more than 28 days to create weekly patterns will provide good prediction performances.

When we look at the performance of the models for multistep prediction, the results indicated that both NARX and MLP models perform significantly better than NAR model. The results also show that MLP models' performances are statistically better than NARX models. We found that NARX with 35-day lead time are the most optimum for multi-step prediction and MLP with 21-day are optimum setting for data set 1 and 35-day for data set 2 . These results clearly demonstrate the effectiveness of using weekly patterns in 
indoor mobility prediction.

In this paper, we also proposed a new measurement to measure indoor mobility based on entropy. The results of the experiments indicated that the Mobility Entropy (ME) can be used to distinguish between elders with different mobility profiles. A person who has high ME means high indoor mobility. From Fig. 4, it can be seen that a person with low frequency of mobility per hour but has high mobility throughout the day (profile 4) has higher ME than a person with high frequency and low dispersal (profile 3 ). When we inspect the summation of IM values, we found that a person with profile 4 has higher IM values than a person with profile 3 as well. This suggests that ME truly reflect indoor mobility of a person. In the second case study where we simulate data to represent elders who have decline in indoor mobility, the results showed that using $\mathrm{ME}$ the decline can be detected. The results also suggested that using $\mathrm{ME}$, an elderly who has a decline in frequency but still maintain the mobility throughout the day (profile 1) will have higher ME comparing to an elderly who has a decline in mobility throughout the day (profile 2). This reflects on human behaviour such that when a person who usually perform mobility throughout the day but suddenly stop should have lower ME comparing to a person who can still carry on indoor mobility but only less often in an hour.

The proposed model and indoor mobility measurement can be used to predict future behaviour to see whether any deterioration in indoor mobility would happen if the elderly carried on with their current mobility behaviour. This would allow intervention in their behavioural routine such that deterioration is prevented or reduced. The proposed work can also be used to capture individual behavioural patterns which are then used to detect abnormal behaviour. For example, a person may normally goes to bed at $11 \mathrm{pm}$, but this may be considered as an abnormal behaviour for a different person. This allows personalized care or services to suit different individuals.

\section{CONCLUSION}

In this paper, we have proposed a prediction model based on indoor mobility using binary sensor information in an AmI environment. The proposed model is developed based on a neural network using weekly patterns. The results of the study show that there is a weekly pattern in human behaviour. Based on the experimental study, using the weekly routine in the model captured the indoor mobility behaviour. However, enough information is required to build the weekly pattern and a recommendation of at least 28 days is suggested. This study also proposed mobility entropy (ME) to measure indoor mobility based on entropy concept. The experimental results demonstrated that $\mathrm{ME}$ is directly linked with indoor mobility values. ME can be used to distinguish between persons with different mobility and as a measurement to see the decline in mobility. For future work, evaluation will be carried out on the proposed work over a longer period of prediction, and techniques to detect changes in behaviour and decline in mobility will be explored.

\section{ACKNOWLEDGMENT}

This project has received funding from Technology Strategy Board (UK) and European Ambient Assisted Living
(AAL) joint programme under the Grant number AAL-20125-239 (iCarer).

\section{REFERENCES}

[1] "World population ageing: 1950-2050," Department of Economic and Social Affairs, United Nations, Tech. Rep., 2002. [Online]. Available: http://www.un.org/esa/population/publications/worldageing19502050/

[2] S. Sutherland and M. Henwood, Carers of elderly people: summary of the background evidence. London: Stationery Office, 1999, vol. 3, pp. 17-28.

[3] B. Najafi, K. Aminian, A. Paraschiv-Ionescu, F. Loew, C. Bula, and P. Robert, "Ambulatory system for human motion analysis using a kinematic sensor: monitoring of daily physical activity in the elderly," Biomedical Engineering, IEEE Transactions on, vol. 50, no. 6, pp. 711723, 2003.

[4] S. Chernbumroong, S. Cang, A. Atkins, and H. Yu, "Elderly activities recognition and classification for applications in assisted living," Expert Systems with Applications, vol. 40, no. 5, pp. 1662-1674, 2013.

[5] T. Barger, D. Brown, and M. Alwan, "Health-status monitoring through analysis of behavioral patterns," Systems, Man and Cybernetics, Part A: Systems and Humans, IEEE Transactions on, vol. 35, no. 1, pp. 22-27, Jan 2005.

[6] S. Mahmoud, A. Lotfi, and C. Langensiepen, "Behavioural pattern identification and prediction in intelligent environments," Applied Soft Computing, vol. 13, no. 4, pp. 1813 - 1822, 2013.

[7] S. Rai and $\mathrm{X}$. Hu, "Behavior pattern detection for data assimilation in agent-based simulation of smart environments," in Web Intelligence (WI) and Intelligent Agent Technologies (IAT), 2013 IEEE/WIC/ACM International Joint Conferences on, vol. 2, Nov 2013, pp. 171-178.

[8] S.-W. Lee, Y. S. Kim, and Z. Bien, "A nonsupervised learning framework of human behavior patterns based on sequential actions," Knowledge and Data Engineering, IEEE Transactions on, vol. 22, no. 4, pp. 479-492, April 2010.

[9] D. Lymberopoulos, T. Teixeira, and A. Savvides, "Detecting patterns for assisted living using sensor networks: A case study," in Sensor Technologies and Applications, 2007. SensorComm 2007. International Conference on, Oct 2007, pp. 590-596.

[10] C. Fernandez-Llatas, J. Garcia-Gomez, J. Vicente, J. Naranjo, M. Robles, J.-M. Benedi, and V. Traver, "Behaviour patterns detection for persuasive design in nursing homes to help dementia patients," in Engineering in Medicine and Biology Society,EMBC, 2011 Annual International Conference of the IEEE, Aug 2011, pp. 6413-6417.

[11] C.-W. Chen, A. Aztiria, and H. Aghajan, "Learning human behaviour patterns in work environments," in Computer Vision and Pattern Recognition Workshops (CVPRW), 2011 IEEE Computer Society Conference on, June 2011, pp. 47-52.

[12] H. Hashidate, H. Shimada, T. Shiomi, M. Shibata, K. Sawada, and N. Sasamoto, "Measuring indoor life-space mobility at home in older adults with difficulty to perform outdoor activities," Journal of geriatric physical therapy, vol. 36, no. 3, pp. 109-114, 2013.

[13] S. Lord, S. F. M. Chastin, L. McInnes, L. Little, P. Briggs, and L. Rochester, "Exploring patterns of daily physical and sedentary behaviour in community-dwelling older adults," Age and Ageing, vol. 40, no. 2, pp. 205-210, 2011.

[14] M. Davis and K. Fox, "Physical activity patterns assessed by accelerometry in older people,' European Journal of Applied Physiology, vol. 100, no. 5, pp. 581-589, 2007.

[15] M. Tinetti and S. Ginter, "The nursing home life-space diameter. a measure of extent and frequency of mobility among nursing home residents." Journal of the American Geriatrics Society, vol. 38, no. 12, pp. 1311-1315, 1990.

[16] R. Clausius, The mechanical theory of heat. Macmillan and Company, 1879.

[17] H. R. Maei, K. Zaslavsky, A. H. Wang, A. P. Yiu, C. M. Teixeira, S. A. Josselyn, and P. W. Frankland, "Development and validation of a sensitive entropy-based measure for the water maze," Frontiers in integrative neuroscience, vol. 3, 2009. 
TABLE III. A COMPARISON BETWEEN THE MODELS PERFORMANCES USING DIFFERENT $l$ LEAD TIME WHERE $H$ IS NUMBER OF HIDDEN NODES, $\alpha$ IS NUMBER OF DELAYS, $\oplus$ IS TRAIN PERFORMANCE, $\ominus$ IS VALIDATION PERFORMANCE AND $\otimes$ IS TEST PERFORMANCE ON DATA SET 1.

\begin{tabular}{|l|c|c|c|c|c|}
\hline Model & $H$ & $\alpha$ (hour) & $\oplus$ & $\ominus$ & $\otimes$ \\
\hline NAR-IM & 5 & 10 & $0.0183 \pm 0.0065$ & $0.0236 \pm 0.0153$ & $0.0284 \pm 0.0064$ \\
NARX-IM-14 & 5 & 1 & $0.0241 \pm 0.0038$ & $0.0283 \pm 0.0090$ & $0.0268 \pm 0.0036$ \\
NARX-IM-21 & 5 & 1 & $0.0220 \pm 0.0024$ & $0.0275 \pm 0.0088$ & $0.0277 \pm 0.0028$ \\
NARX-IM-28 & 10 & 10 & $0.0150 \pm 0.0063$ & $0.0216 \pm 0.0111$ & $0.0267 \pm 0.0045$ \\
NARX-IM-35 & 30 & 10 & $0.0107 \pm 0.0052$ & $0.0199 \pm 0.0096$ & $0.0266 \pm 0.0033$ \\
MLP-IM-14 & 5 & - & $0.0152 \pm 0.0070$ & $0.0184 \pm 0.0079$ & $0.0204 \pm 0.0063$ \\
MLP-IM-21 & 5 & - & $0.0178 \pm 0.0088$ & $0.0165 \pm 0.0086$ & $0.0197 \pm 0.0084$ \\
MLP-IM-28 & 15 & - & $0.0130 \pm 0.0042$ & $0.0170 \pm 0.0093$ & $0.0160 \pm 0.0027$ \\
MLP-IM-35 & 10 & - & $0.0127 \pm 0.0048$ & $0.0153 \pm 0.0082$ & $0.0182 \pm 0.0026$ \\
\hline
\end{tabular}

TABLE IV. A STATISTICAL RESUlts (P VALUE) FOR MODELS COMPARISON ON DATA SET 1.

\begin{tabular}{|l|c|c|c|c|c|c|c|c|c|}
\hline Model & NAR-IM & NARX-IM-14 & NARX-IM-21 & NARX-IM-28 & NARX-IM-35 & MLP-IM-14 & MLP-IM-21 & MLP-IM-28 & MLP-IM-35 \\
\hline NAR-IM & - & 0.163 & 0.553 & 0.070 & 0.327 & 0.000 & 0.000 & 0.000 & 0.000 \\
NARX-IM-14 & 0.163 & - & 0.013 & 0.502 & 0.935 & 0.000 & 0.000 & 0.000 & 0.000 \\
NARX-IM-21 & 0.553 & 0.013 & - & 0.042 & 0.055 & 0.000 & 0.000 & 0.000 & 0.000 \\
NARX-IM-28 & 0.070 & 0.502 & 0.042 & - & 0.682 & 0.000 & 0.000 & 0.000 & 0.000 \\
NARX-IM-35 & 0.327 & 0.935 & 0.055 & 0.682 & - & 0.000 & 0.000 & 0.000 & 0.000 \\
MLP-IM-14 & 0.000 & 0.000 & 0.000 & 0.000 & 0.000 & - & 0.045 & 0.000 & 0.042 \\
MLP-IM-21 & 0.000 & 0.000 & 0.000 & 0.000 & 0.000 & 0.045 & - & 0.010 & 0.935 \\
MLP-IM-28 & 0.000 & 0.000 & 0.000 & 0.000 & 0.000 & 0.000 & 0.010 & - & 0.000 \\
MLP-IM-35 & 0.000 & 0.000 & 0.000 & 0.000 & 0.000 & 0.042 & 0.935 & 0.000 & - \\
\hline
\end{tabular}

TABLE V. A COMPARISON BETWEEN THE MODELS PERFORMANCES USING DIFFERENT $l$ LEAD TIME WHERE $H$ IS NUMBER OF HIDDEN NODES, $\alpha$ IS NUMBER OF DELAYS, $\oplus$ IS TRAIN PERFORMANCE, $\ominus$ IS VALIDATION PERFORMANCE AND $\otimes$ IS TEST PERFORMANCE ON DATA SET 2.

\begin{tabular}{|l|c|c|c|c|c|}
\hline Model & $H$ & $\alpha$ (hour) & $\oplus$ & $\ominus$ & $\otimes$ \\
\hline NAR-IM & 5 & 2 & $0.0501 \pm 0.0113$ & $0.0485 \pm 0.0157$ & $0.0569 \pm 0.0176$ \\
NARX-IM-14 & 5 & 3 & $0.0311 \pm 0.0079$ & $0.0326 \pm 0.0076$ & $0.0373 \pm 0.0056$ \\
NARX-IM-21 & 5 & 1 & $0.0331 \pm 0.0048$ & $0.0316 \pm 0.0088$ & $0.0355 \pm 0.0050$ \\
NARX-IM-28 & 5 & 1 & $0.0309 \pm 0.0026$ & $0.0290 \pm 0.0088$ & $0.0337 \pm 0.0040$ \\
NARX-IM-35 & 10 & 9 & $0.0232 \pm 0.0088$ & $0.0304 \pm 0.0079$ & $0.0352 \pm 0.0059$ \\
MLP-IM-14 & 10 & - & $0.0212 \pm 0.0032$ & $0.0251 \pm 0.0126$ & $0.0288 \pm 0.0031$ \\
MLP-IM-21 & 5 & - & $0.0212 \pm 0.0094$ & $0.0224 \pm 0.0144$ & $0.0271 \pm 0.0068$ \\
MLP-IM-28 & 5 & - & $0.0226 \pm 0.0098$ & $0.0231 \pm 0.0140$ & $0.0265 \pm 0.0086$ \\
MLP-IM-35 & 5 & - & $0.0191 \pm 0.0018$ & $0.0207 \pm 0.0082$ & $0.0236 \pm 0.0027$ \\
\hline
\end{tabular}

TABLE VI. A STATISTICAL RESUlTS (P VALUE) FOR MODELS COMPARISON ON DATA SET 2.

\begin{tabular}{|l|c|c|c|c|c|c|c|c|c|}
\hline Model & NAR-IM & NARX-IM-14 & NARX-IM-21 & NARX-IM-28 & NARX-IM-35 & MLP-IM-14 & MLP-IM-21 & MLP-IM-28 & MLP-IM-35 \\
\hline NAR-IM & - & 0.000 & 0.000 & 0.000 & 0.000 & 0.000 & 0.000 & 0.000 & 0.000 \\
NARX-IM-14 & 0.000 & - & 0.078 & 0.001 & 0.160 & 0.000 & 0.000 & 0.000 & 0.000 \\
NARX-IM-21 & 0.000 & 0.078 & - & 0.075 & 0.768 & 0.000 & 0.000 & 0.000 & 0.000 \\
NARX-IM-28 & 0.000 & 0.001 & 0.075 & - & 0.157 & 0.000 & 0.000 & 0.000 & 0.000 \\
NARX-IM-35 & 0.000 & 0.160 & 0.768 & 0.157 & - & 0.000 & 0.000 & 0.000 & 0.000 \\
MLP-IM-14 & 0.000 & 0.000 & 0.000 & 0.000 & 0.000 & - & 0.000 & 0.000 & 0.000 \\
MLP-IM-21 & 0.000 & 0.000 & 0.000 & 0.000 & 0.000 & 0.000 & - & 0.154 & 0.000 \\
MLP-IM-28 & 0.000 & 0.000 & 0.000 & 0.000 & 0.000 & 0.000 & 0.154 & - & 0.001 \\
MLP-IM-35 & 0.000 & 0.000 & 0.000 & 0.000 & 0.000 & 0.000 & 0.000 & 0.001 & - \\
\hline
\end{tabular}

TABLE VIII. A STATISTICAL RESUlts (P VALUE) FOR MODELS COMPARISON ON DATA SET 1 ON MULTI-STEP PREDICTION.

\begin{tabular}{|c|c|c|c|c|c|c|c|c|c|}
\hline Model & NAR-IM & NARX-IM-14 & NARX-IM-21 & NARX-IM-28 & NARX-IM-35 & MLP-IM-14 & MLP-IM-21 & MLP-IM-28 & MLP-IM-35 \\
\hline NAR-IM & - & 0.000 & 0.000 & 0.000 & 0.000 & 0.000 & 0.000 & 0.000 & 0.000 \\
\hline NARX-IM-14 & 0.000 & - & 0.390 & 0.000 & 0.000 & 0.000 & 0.000 & 0.000 & 0.000 \\
\hline NARX-IM-21 & 0.000 & 0.390 & - & 0.000 & 0.000 & 0.000 & 0.000 & 0.000 & 0.000 \\
\hline NARX-IM-28 & 0.000 & 0.000 & 0.000 & - & 0.000 & 0.000 & 0.000 & 0.000 & 0.000 \\
\hline NARX-IM-35 & 0.000 & 0.000 & 0.000 & 0.000 & - & 0.000 & 0.000 & 0.000 & 0.000 \\
\hline MLP-IM-14 & 0.000 & 0.000 & 0.000 & 0.000 & 0.000 & & 0.000 & 0.000 & 0.000 \\
\hline MLP-IM-21 & 0.000 & 0.000 & 0.000 & 0.000 & 0.000 & 0.000 & - & 0.000 & 0.000 \\
\hline MLP-IM-28 & 0.000 & 0.000 & 0.000 & 0.000 & 0.000 & 0.000 & 0.000 & - & 0.000 \\
\hline MLP-IM-35 & 0.000 & 0.000 & 0.000 & 0.000 & 0.000 & 0.000 & 0.000 & 0.000 & - \\
\hline
\end{tabular}

TABLE X. A STATISTICAL RESUlts (P VALUE) FOR MODELS COMPARISON ON DATA SET 2 ON MULTI-STEP PREDICTION.

\begin{tabular}{|l|c|c|c|c|c|c|c|c|c|}
\hline Model & NAR-IM & NARX-IM-14 & NARX-IM-21 & NARX-IM-28 & NARX-IM-35 & MLP-IM-14 & MLP-IM-21 & MLP-IM-28 & MLP-IM-35 \\
\hline NAR-IM & - & 0.000 & 0.000 & 0.000 & 0.000 & 0.000 & 0.000 & 0.000 & 0.000 \\
NARX-IM-14 & 0.000 & - & 0.000 & 0.001 & 0.000 & 0.000 & 0.000 & 0.000 & 0.000 \\
NARX-IM-21 & 0.000 & 0.000 & - & 0.000 & 0.000 & 0.000 & 0.000 & 0.000 & 0.000 \\
NARX-IM-28 & 0.000 & 0.001 & 0.000 & - & 0.000 & 0.000 & 0.000 & 0.000 & 0.000 \\
NARX-IM-35 & 0.000 & 0.000 & 0.000 & 0.000 & - & 0.000 & 0.000 & 0.000 & 0.000 \\
MLP-IM-14 & 0.000 & 0.000 & 0.000 & 0.000 & 0.000 & - & 0.000 & 0.000 & 0.000 \\
MLP-IM-21 & 0.000 & 0.000 & 0.000 & 0.000 & 0.000 & 0.000 & - & 0.000 & 0.000 \\
MLP-IM-28 & 0.000 & 0.000 & 0.000 & 0.000 & 0.000 & 0.000 & 0.000 & - & 0.000 \\
MLP-IM-35 & 0.000 & 0.000 & 0.000 & 0.001 & 0.000 & 0.000 & 0.000 & 0.000 & - \\
\hline
\end{tabular}

